\title{
Progressive encephalopathy with leukodystrophy due to DECR deficiency
}

INSERM

\section{Source}

INSERM. (1999). Orphanet: an online rare disease and orphan drug data base.

Progressive encephalopathy with leukodystrophy due to DECR deficiency.

ORPHA:431361

Progressive encephalopathy with leukodystrophy due to DECR deficiency is a rare mitochondrial disease, which presents with neonatal hypotonia, central nervous system abnormalities (ventriculomegaly, corpus callosum hypoplasia, cerebellar atrophy), acquired microcephaly, failure to thrive, developmental delay and intermittent lactic acidosis provoked by catabolic stress (e.g. infection). Hyperlysinemia and elevated C10:2 carnitine can be detected in plasma. Later on, epilepsy, cerebellar ataxia, renal tubular acidosis, severe encephalopathy, dystonia, spastic quadriplegia and other complications may develop. 